\title{
In-Situ Operation of Nanoelectronic Devices
}

\author{
John Cumings*, Todd Brintlinger*, Kamal Baloch**, and Yi Qi* \\ *Department of Materials Science and Engineering, University of Maryland, College Park, MD, \\ USA \\ **Institute for Physical Science and Technology, University of Maryland, College Park, MD, USA
}

The interactions of electrical current and materials structure are of fundamental importance to the future of nanoscale systems. Subtle changes in nanostructure can induce marked changes in the electrical transport properties, and an electrical current can, in turn, induce its own changes in a system. These correlations between nanostructure and electrical transport are most important and complex as device sizes approach the nanoscale, and, unfortunately, this is the very realm where traditional techniques for observing device dynamics fail.

Traditionally, the dynamics of electrical and mechanical devices are studied by video-rate light microscopy techniques. These imaging techniques are limited by the wavelength of light and are thus ineffective on nanoscale systems. Although there are a few key instruments, like the STM and AFM, that have enabled the early development of nanosystems, they provide only a limited view, due to a slow imaging speed. Transmission electron microscopy (TEM) is a natural candidate for extending a larger class of studies into the nanoscale realm, but conventionally TEM studies of devices have focused only on uncovering the materials' structure before or after device operation. At the University of Maryland, we are building up a research program dedicated to studying the structure and performance of nanoscale devices during device operation. In comparison with scanned-probe microscopies, TEM images can be acquired a thousand times faster, at video rates. This means that, practically speaking, an experimenter can accomplish in about a day the same scope of experiments on a nanoscale device that would require a year's worth of study using a scanned probe technique, such as STM or AFM.

There are two key tools that make this area of research possible. The first is a nano-manipulation stage. This is essentially just an STM that can be operated inside a TEM [1]. It allows electrical probing and deformation of nanomaterials during microscopic investigations [2-5]. Using this, it is possible to fabricate devices in-situ and observe their operation at the same time. The second tool is a reliable multi-contact electrical measurement specimen holder. We have developed the techniques for performing reliable lithography (both photolithography and electron-beam lithography) on commercially available silicon nitride membranes. Devices fabricated on these substrates can be investigated in any TEM specimen holder during the fabrication process and then can be transferred to the electrical measurement holder for in-situ observations of the device operation.

These capabilities enable a wide variety of new research areas. We have already used this approach for the fabrication of ultra-fast nanoscale heating strips $(\sim 10 \mu \mathrm{s}$ response time). These heating strips also generate very strong temperature gradients that may be used for imaging how material's structure evolves in a strong gradient, for imaging the non-equilibrium structure of a material in response to very fast temperature changes, or for performing in-situ combinatorial processing studies by annealing a material in-situ in a temperature gradient. These heating strips are in-turn also being used as a control device for the development of nanoscale thermal imaging techniques. These 
thermal imaging techniques will ultimately be used to test models of dissipative and non-dissipative transport in nanoscale electronic devices.

\section{References:}

[1] E. A. Stach, et al. Microsc. Microanal., 7(6), p.507 (2001)

[2] J. Cumings, P. G. Collins, and A. Zettl, Nature 406, 586 (2000)

[3] J. Cumings and A. Zettl, Phys. Rev. Lett. 93, 086801 (2004)

[4] J. Cumings, A. Zettl, M. R. McCartney, and J. C. H. Spence, Phys. Rev. Lett. 88, 056804 (2002)

[5] J. Cumings, A. Zettl, and M. R. McCartney, Microsc. Microanal. 10, 420 (2004)
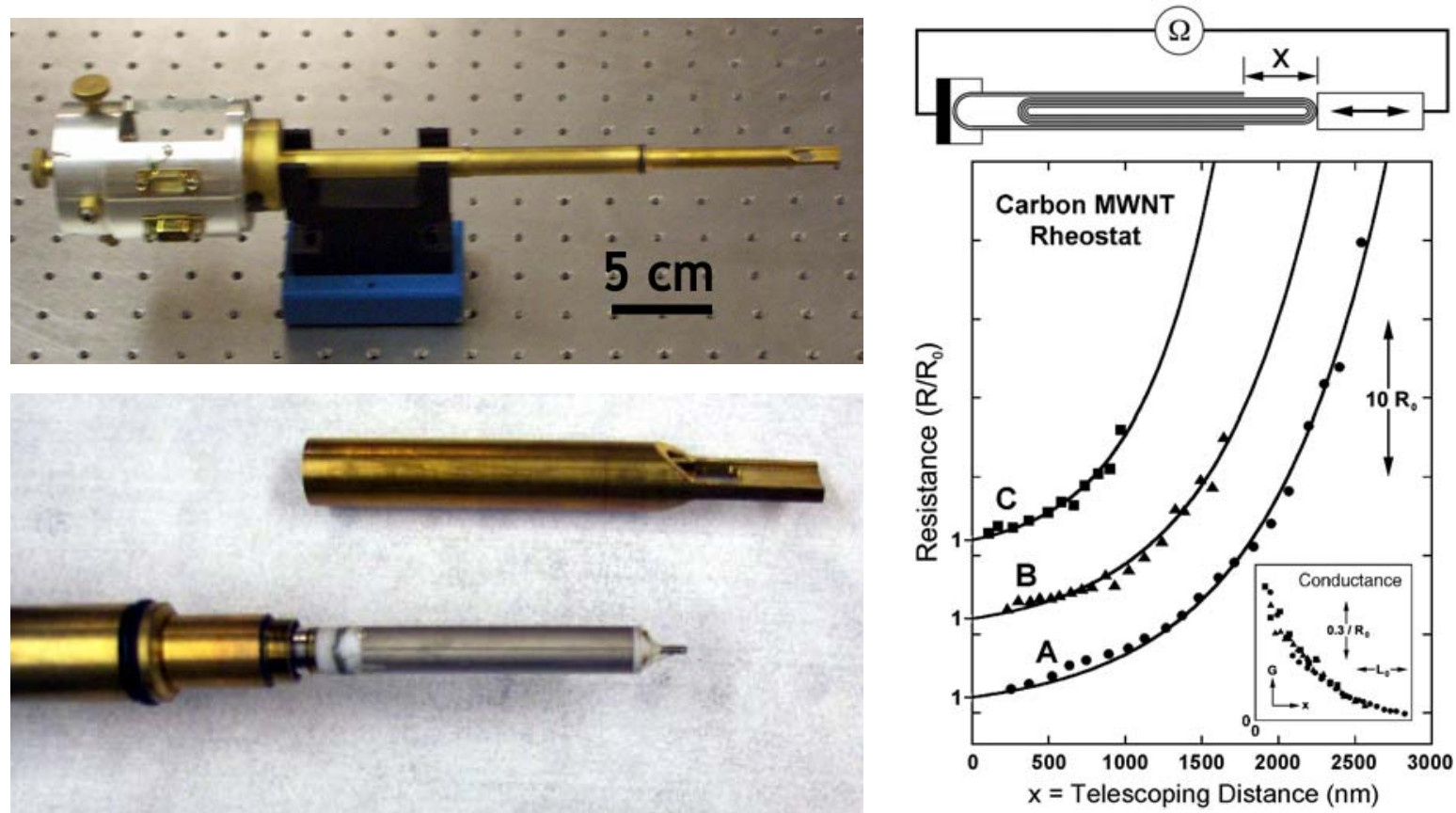

FIG. 1. A nanomanipulation stage constructed for studying nanodevices formed in-situ inside a TEM. On the right is shown one specific device, a nanoscale variable resistor formed from a multiwall carbon nanotube (from [3]).

FIG. 2. Top: an electrical measurement stage for measuring devices fabricated on standard silicon nitride membranes. Bottom left: a silicon nitride membrane from SPI, inc (image from SPI website). Bottom middle: a nanoscale device fabricated on one of these $2.6 \mathrm{~mm}$ silicon chips using both photolithography and electron-beam lithography. Bottom right: A device as it is electrically contacted inside the TEM holder.

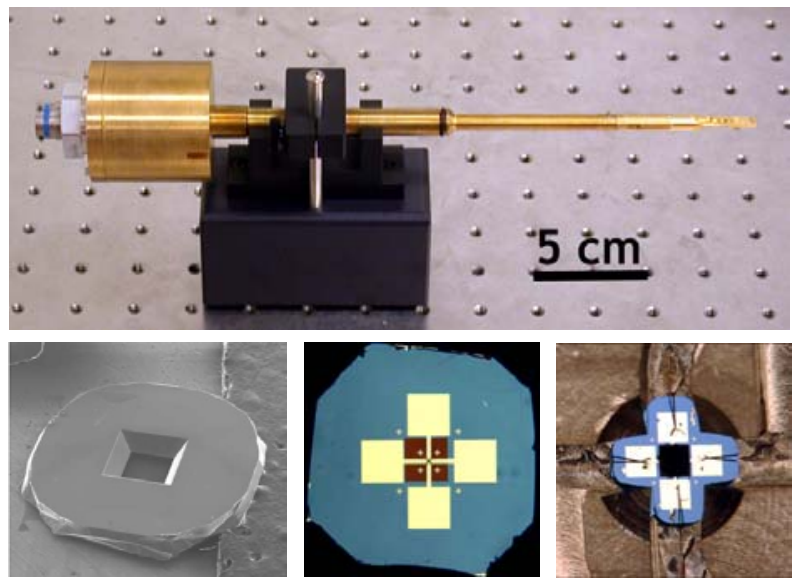

